\title{
Mendelian and non-Mendelian inheritance of newly-arisen chromosome rearrangements
}

\author{
A. S. Wilby and \\ J. S. Parker
}

School of Biological Sciences, Queen Mary College, University of London, Mile End Road, London E1 4NS, U.K.

Seven centric shifts and three reciprocal interchanges, all newly-arisen in natural populations, have been tested for their inheritance in the dioecious flowering plant Rumex acetosa. In backcrosses between the heterozygote and standard plants transmissions ranged from 0.36 to 0.85 per gamete for the novel chromosome. The inheritance of only four rearrangements correspond to Mendelian expectations while others exhibited either drive or drag. Drive was observed both through the egg and through the pollen indicating heterogeneity of mechanisms in the generation of nonMendelian patterns of inheritance. This suggests that accumulation may play a significant role in the establishment of chromosomal variants in natural populations.

\section{INTRODUCTION}

Closely-related species usually differ in their chromosome constitutions (White, 1978). Many of these differences, when in the hybrid condition, depress the fertility of the F1 by deranging the orderly progress of meiosis. Reciprocal translocations in particular disturb regular disjunction such that the overall fertility is reduced to about 50 per cent (Darlington, 1965). Since the fixed chromosome differences which are commonplace between species have arisen within species in a heterozygous condition then for establishment they must have passed through a fertility trough in the production of the homokaryotype. The question arises then as to how fixation has occurred, and on such a massive scale, in the face of negative heterosis.

One possibility is that novel karyotypes become established in locally-fragmented demes with small population sizes by inbreeding. This may be followed by movement of the new homokaryotype into vacant areas following periodic extinctions (Simberloff, 1974). Spread of the homokaryotype avoids much of the negative heterosis which would accompany structural heterozygosity (Lande, $1979,1985)$. This scheme may be applicable to the plant genus Clarkia where chromosome repatterning has occurred many times in semi-arid regions peripheral to the more mesic areas of California (Lewis, 1973).
One factor which may aid the spread of a novel chromosome rearrangement through a population is meiotic drive. Hedrick (1981) has shown that both meiotic drive alone, and in combination with genetic drift, can be significant in the establishment of chromosome rearrangements. Even a small amount of drive can lead to a great increase in the probability of fixation of a new variant which shows heterozygous disadvantage.

Alleles which show drive would not be expected to remain in a polymorphic condition in a species but to proceed to fixation, except in situations where one homozygote is subject to massive selection such as Segregation Distorter in Drosophila (Hiraizumi et al., 1960). Drive, however, might be assessed from the recovery of gametes from interspecific hybrids since species may be fixed for driving alleles. Interpretation may perhaps be obscured by problems of inviability and selective recovery of offspring unrelated to driving alleles. A study of three interspecific Drosophila hybrids by Coyne (1985) showed no evidence of fixed differences of driving alleles. He points out that massive numbers of offspring would have to be scored to detect small amounts of drive although these could still be significant in leading to fixation.

Recently a supernumerary segment polymorphism showing drive has been found in the plant Rumex acetosa. Egg transmission from 

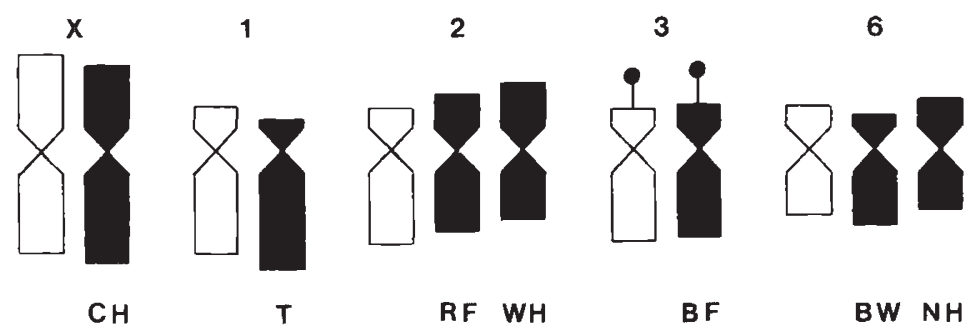

Figure 1 Centric shifts in chromosomes $X, 1,2,3$ and 6 of Rumex acetosa. The standard chromosomes are shown in outline. The population origin of each novel chromosome is indicated (see table 1).

heterozygotes was about 0.63 rather than the expected 0.5 (Wilby and Parker, 1987). Attendent decline in fitness has not been detected although there is some indication of a deficiency of homozygotes in populations. During studies of the cytological structure of natural populations of this species a large number of chromosome mutants have been recovered, all as heterozygotes. If meiotic drive is a potent force in the fixation of chromosome rearrangements then at least some of these mutants should show deviations from strict Mendelian inheritance. In this paper the patterns of inheritance of 10 rearrangements -7 centric shifts and 3 reciprocal interchanges-are examined, mostly in backcrosses. Overall accumulation, overall loss and standard Mendelian inheritance has been demonstrated indicating that drive may be of significance in fixation, or indeed extinction, of newly-arisen chromosome rearrangements.

\section{MATERIALS AND METHODS}

The mutants were found in seed samples from natural populations of Rumex acetosa. With one exception, they were recovered as single individuals in samples of at least 25 plants per population. In each case the rest of the half-sib family scored carried a standard complement. Thus the mutants were probably collected at or soon after their generation and certainly before polymorphic levels were reached. One rearrangement-an $X / 2$ reciprocal interchange-was found as single individuals in two families in a small, isolated population (Bury Hill).

Mitotic chromosome preparations were made by Feulgen-staining of root-tips following pretreatment with 0.05 per cent colchicine and fixation in $1: 3$ acetic alcohol. Meiotic studies were carried out on buds fixed in Carnoy and stained in 2 per cent acetocarmine.
Most crosses were between the heterozygote and a standard plant, a backcross. Behaviour of two rearrangements was also examined in crosses involving individuals of the backcross generation. In most cases then a $1: 1$ ratio is expected in the progeny.

\section{RESULTS}

\section{The basic karyotype}

Rumex acetosa has $2 n=12+X X$ in females and $2 n=12+X Y 1 Y 2$ in males. The sex-chromosomes are the largest members of the complement, about 50 per cent longer than autosome 1 . The $X$ is metacentric while $Y 1$ and $Y 2$ are very variable in centromere location (Wilby and Parker, 1986, 1987). The autosomes are highly acrocentric with arm-ratios between $1: 2.28$ and $1: 4.63$ and are individually recognisable. The acrocentricity of the complement aids detection of rearrangements and during screening of mitotic complements of natural population plants 27 structural variants have been found in 1254 individuals (Wilby, unpublished).

\section{The chromosome rearrangements}

Seven centric shifts, probably resulting from pericentric inversion, of chromosomes $1,2,3,6$ and $X$ have been studied, two each in chromosomes 2 and 6 . Three gave more acrocentric chromosomes $(X, 1$ and 6$)$ and the rest more metacentric (fig. 1).

Three reciprocal interchanges were also examined: $X / 2, X / 3$ and $5 / 6$. In the $X / 2$ exchange, the $X$ product has a shortened $Y 2$ pairing arm and a 2 with an increased long arm. In the $X / 3$ exchange, one break point is in the $Y 1$ pairing arm of the $X$ and the other through the satellite region of the nucleolar-organiser chromosome 3 . It is unclear if this is a reciprocal exchange but unbalanced transmissions indicate that no 


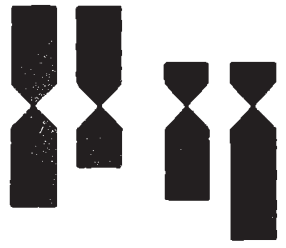

$$
x \quad x^{2} \quad 2 \quad 2^{x}
$$
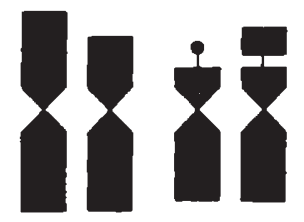

$\mathrm{x} \quad \mathrm{x}^{3} \quad 3 \quad 3^{\mathrm{x}}$

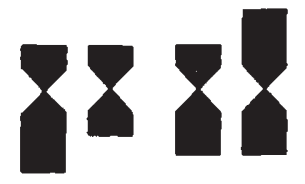

$5 \quad 5^{6} \quad 6 \quad 6^{5}$

Figure 2 Chromosome complements of the heterozygous interchanges $X / 2, X / 3$ and $5 / 6$ in $R$. acetosa.

essential information has been transferred to the $X$ from 3 . The final interchange- $5 / 6$-had breakpoints in the short-arm of 6 and long-arm of 5 giving a short metacentric 5 and a long 6 (fig. 2).

Inheritance of each rearrangement has been examined using the individual in which the rearrangement was first identified and centric shift 1 and the $X / 2$ interchange have also been studied in crosses involving the offspring. For the remainder, therefore, only one direction of crossing is reported.

\section{The patterns of inheritance}

\section{(a) Centric shifts}

Of the nine crosses only four conformed to Mendelian expectations: four showed excess transmission of the novel chromosome and one overall loss (table 1).

Inheritance of chromosome 1 centric shift (fig. 3 ), detected in a female, was also tested through pollen and eggs in the offspring generation. In the initial cross $67 / 102$ offspring carried the novel chromosome, a transmission rate of 0.66 and a deviation from expectation significant at the 1 per cent level (table 1). Transmission rate of the female offspring was 0.65 but non-significant with a sample size of only 40 . By contrast, there was a significant loss through the pollen $(\bar{k}=0.31 ; P<$ $0 \cdot 05)$.

Two distinguishable shifts of chromosome 2 have been examined in males from the Rye Foreign and Willersey Hill populations. Remarkably, both show enhanced inheritance with transmission values of 0.69 (RF2) and 0.85 (WH2), significantly different from expectation at the 5 per cent and $0 \cdot 1$ per cent levels respectively.

The shift in the nucleolar-organiser chromosome 3 had a transmission of $0 \cdot 4$. Even with a sample size of 75 this is not a significant deviation from expectation.

Seven different shifts of chromosome 6 have been found in natural populations. Two have been examined, one more acrocentric (BW6) and the other more metacentric (N6) than standard, and both conform to Mendelian expectations.

Finally, the acrocentric $X$ gave a transmission frequency, through the egg of 0.78 , a deviation significant at the 1 per cent level.

\section{(b) Reciprocal interchanges}

The $X / 2$ interchange (fig. 4 ), found in two females from the Bury Hill population, showed enhanced

Table 1 The patterns of inheritance of centric shifts in Rumex acetosa. All crosses are backcrosses and the mean gametic transmission of the novel chromosome is given $(\tilde{k})$. The superscript ${ }^{2}$ indicates that the cross involves one of the offspring of the original isolate. Population locations are given in Wilby and Parker, 1987

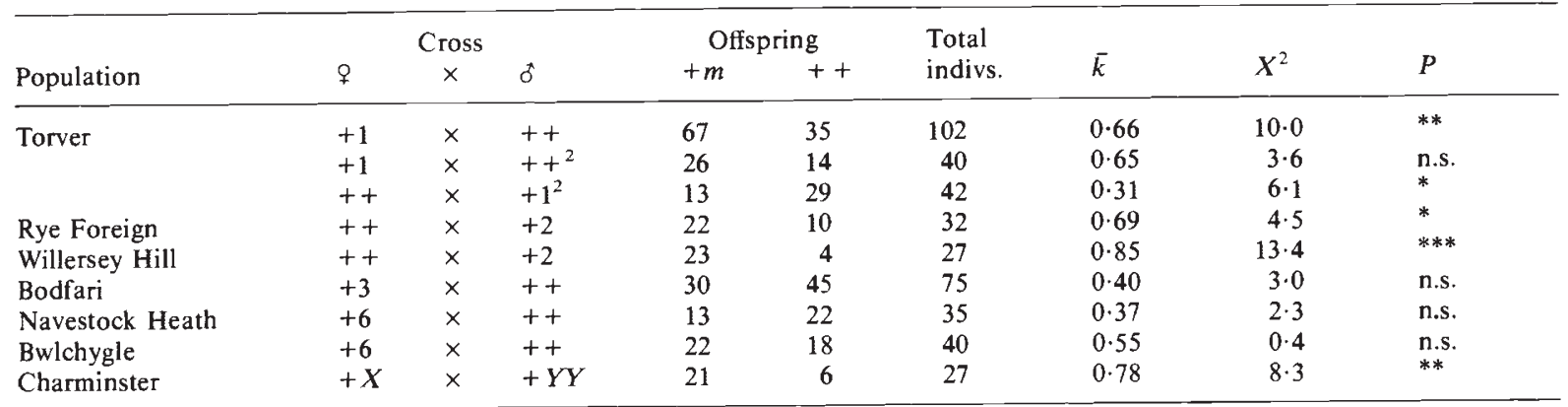




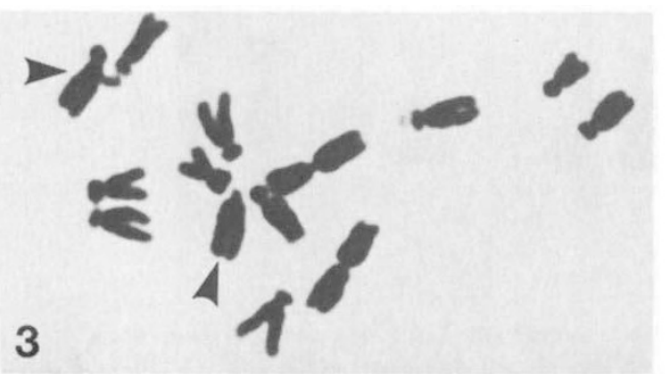

Figure 3 The mitotic complement of a female plant of $R$. acetosa heterozygous for the centric shift on chromosome 1. Both members of pair 1 are arrowed.

transmission of $0.64 \quad(P<0 \cdot 01)$. Three further crosses have been carried out using the offspring: (i) female heterozygote $\times$ standard male, (ii) standard female $\times$ hemizygote male, and (iii) heterozygote $\times$ hemizygote. In crosses (i) and (ii) transmission was 0.59 and 0.63 respectively. Both are non-significant but sample sizes are rather low. In cross (iii) transmission through both gametes can be assessed. Pollen transmission is again high $(\vec{k}=$ $0.72 ; P<0.05)$, but egg transmission shows a close approximation to the expected. Thus there is no indication of the accumulation which characterised the original female, indicating that factors other than chromosome structure itself influence transmission.

All the products recovered from the $X / 2$ interchange are balanced but behaviour of the $X / 3$ interchange is very different. In heterozygotes three types of functional egg are formed in roughly equal proportions-X $X, X^{3} 3^{X}$, and $X 3^{X} \cdot X^{3} 3$ has not been recovered. Thus about one third of offspring are unbalanced, trisomics for the translocated portion of the $X$. The satellite region of chromosome 3 is clearly not essential for survival. If balanced products only are considered there is equal transmission of gametes but considering individual

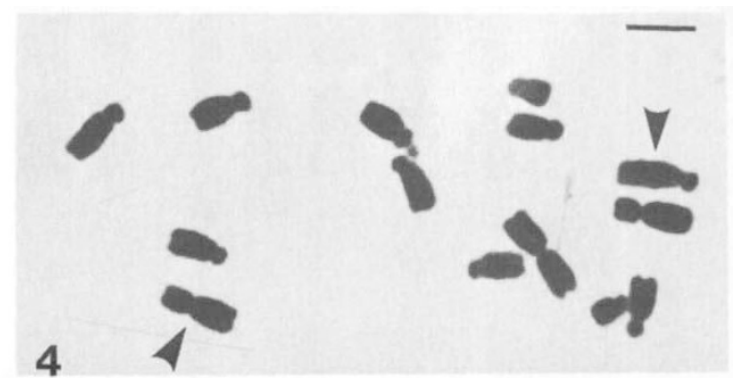

Figure 4 Female plant of $R$. acetosa heterozygous for the $X / 2$ interchange. Chromosomes $X^{2}$ and $2^{X}$ are arrowed. Bar represents $5 \mu \mathrm{m}$.

chromosomes $X$ and $3^{X}$ are recovered twice as frequently as $X^{3}$ and 3 .

The autosomal translocation $5 / 6$ was found in a male. Through the pollen, transmission is only 0.36 but differs non-significantly from the expected.

\section{DISCUSSION}

The theoretical importance of meiotic drive in the establishment of chromosomal rearrangements has frequently been proposed (White, 1978; Hedrick, 1980). Evidence for the unequal recovery of gametes or zygotes carrying rearrangements in natural populations is, however, remarkably sparse. One possible case has been reported in the grasshopper Vandiemenella by White (1978). In the Vandiemenella species group a narrow hybrid zone occurs between the presumptive parental species and a parapatric one which differs by a chromosome rearrangement. Transmission of the standard and rearranged chromosomes from natural hybrids gives an enhanced recovery of the standard type. Clearly if drive had been implicated in the fixation of the novel chromosome then this,

Table 2 Inheritance patterns of two interchange heterozygotes in Rumex acetosa. Crosses of offspring of the original isolate indicated by the superscript ${ }^{2}$

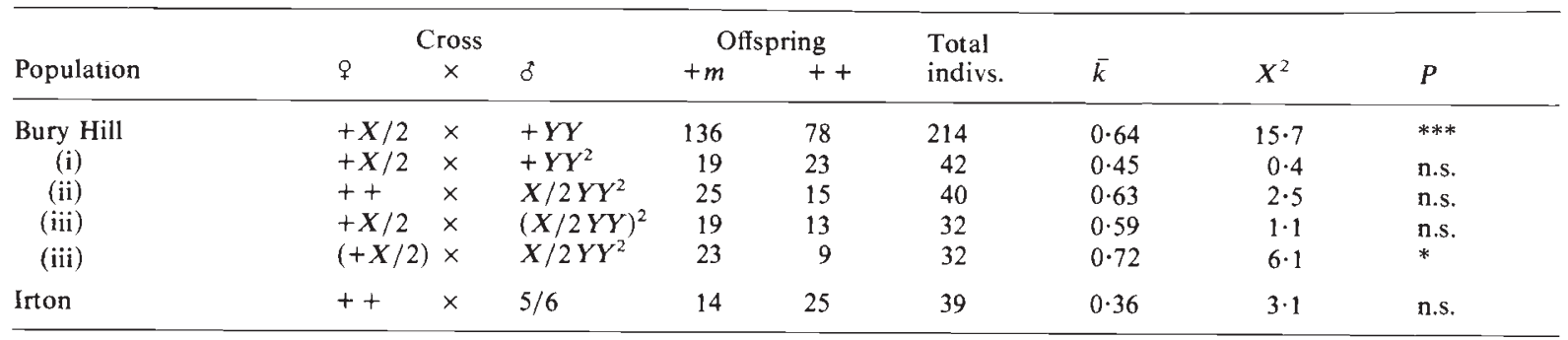


Table 3 Inheritance of an $X / 3$ interchange which gives a high frequency of viable unbalanced gametes through the egg

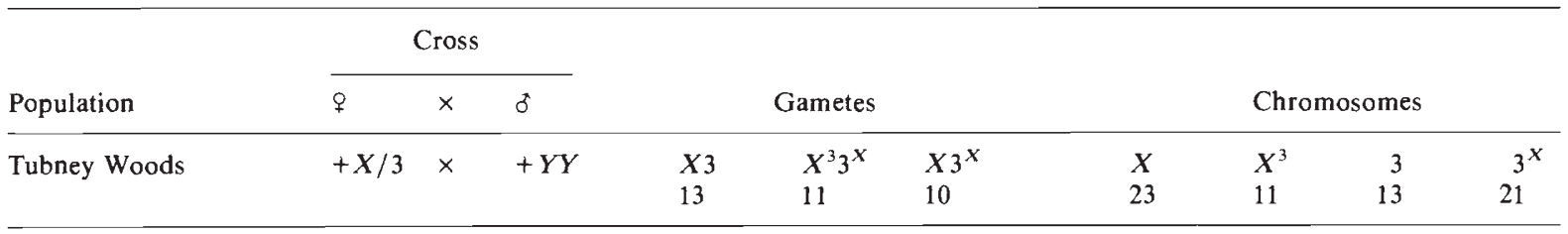

rather than the standard, should have been in excess. The interpretation of these data, however, relies on the correct assessment of the evolutionary relationships of this species pair.

The data on transmission reported in this paper concern rearrangements which have been tested in the generation in which they arose or at most within a few generations of their origin. Thus there should have been no genetic modification of meiotic behaviour patterns as may well have happened in polymorphic situations associated with many Bchromosome systems (Jones and Rees, 1982). It is evident that in this small sample of newly arisen rearrangements behaviour is variable with strict Mendelian inheritance, overall accumulation and overall loss. Although sample sizes are generally not very large, deviations significant at the 1 per cent level have been found. Indeed the data suggest that with larger samples there would be even greater heterogeneity in transmission.

Several distinct mechanisms must be involved in the generation of these deviations from Mendelian expectations. Firstly transmission mechanisms through pollen and eggs must differ. In the female, since only one of the four meiotic products is functional, there is the possibility of directed disjunction to one particular pole. Evidence for spindle asymmetry during meiosis-I and consequent segregation distortion of a large B-chromosome has been given by Hewitt (1976) for the grasshopper Myrmeleotetix maculatus and in Lilium callosum univalent B-chromosomes are observed more frequently on the side of the spindle directed towards the functional micropylar end of the embryo sac (Kayano, 1957).

The excess EMC transmission of the $X / 2$ exchange is associated with a decline in fertility to about 50 per cent. Excess transmission and fertility decline has also been reported for T6-9 in Zea mays by Dempsey (1961). She ascribed this to the formation of trivalent + univalent configurations in which the univalent was usually one of the non-exchange chromosomes and this suffered loss during meiosis. Studies of female meiosis are clearly required in Rumex. On the male side the most frequent configuration is a bivalent $X-Y_{1}$ plus a trivalent $Y 2-2^{X}-2$ in convergent orientation (Wilby, unpublished) which will give equal numbers of the two balanced gametes.

Two mutants showed excess transmission through the pollen. A general mechanism is suggested by considering the behaviour of the $X / 2$ interchange. Most offspring of the $X / 2$ hemizygote male are female and in crosses to standard females these must all be interchange heterozygotes. Excess female production is found normally in natural populations although only with a ratio of $1 \cdot 2: 1$. Under conditions of high pollen density, however, the distortion may be as high as $8: 1$ (Wilby, unpublished). This phenomenon (certation) was first discovered by Correns (1928) who proposed that under high pollination density competition in the style favours $X$-bearing rather than $Y$-bearing pollen-tubes. It is possible that tube-competition is implicated in the transmission of other crosses with the mutants of chromosome 2 favoured over the standard chromosomes. Presumably this would result from chance linkage of genes to the repositioned centromere. Such genes, however, need not be located within the limits of the presumptive inverted regions since with the strict localisation of chiasmata which is found in male Rumex acetosa (Wilby and Parker, 1986) even distal loci may be tightly linked to the centromere. If this is the case the centric shift may act merely as a chromosome marker.

Thus in $R$. acetosa spontaneous chromosome mutants conform to Mendelian expectations only a proportion of the time and they may exhibit overall accumulation or overall loss. If this pattern is repeated in natural populations newly-arisen mutants may be driven to fixation even against a fertility decline or, as pointed out by White (1978), may drive to extinction. Is drive or drag intrinsic to the structure of the chromosome or is it dependent on the background genotype? The evidence of two generations for the chromosome 1 shift and the $X / 2$ interchange points in opposite directions. Even if the inheritance distortion is transient, however, its effects should be shown by the loci carried on that chromosome. Non-random allelic transmission in plants is commonly observed 
(Grant, 1975), usually through the pollen, and distortion may disappear in subsequent generations (Wendel et al., 1987).

The $X / 2$ exchange should prove particularly informative in assessing the role of drive in the establishment of spontaneous rearrangements which show negative heterosis. This interchange was found amongst the progeny of two females in an isolated population which contained less than one hundred individuals. Thus not only does the rearrangement show drive through both eggs and pollen but it has arisen in a small, relatively inbred deme. From the theoretical studies of both Lande (1979) and Hedrick (1980) this rearrangement should have a high probability of sweeping to fixation despite the 50 per cent decline in fertility associated with it. The population will be monitored over successive generations to assess the change in frequency of the rearrangement.

\section{REFERENCES}

CORRENS, C. 1928. Bestimmung, Vererbung und Verteilung des Geschlechtes bei den hoheren Pflanzen. Handb. der Vererb., 2, $1-138$.

COYNE, J. A. 1986. Meiotic segregation and male recombination in interspecific hybrids of Drosophila. Genetics, 114, 485494.

DARLINGTON, C. D. 1965. Cytology. J. \& A. Churchill, London.

DEMPSEY, E. M. 1961. Crossing-over and segregation in plants heterozygous for T6-9b. Maize Genet. Coop. Newsletter, 35, $62-63$.
GRANT, v. 1975. Genetics of flowering Plants. Columbia University Press.

HEDRICK, P. W. 1980. The establishment of chromosomal variants. Evolution, 35, 322-332.

HEWITT, G. M. 1976. Meiotic drive for B-chromosomes in the primary oocytes of Myrmeleotettix maculatus (Orthoptera: Acrididae). Chromosoma, 56, 381-391.

HIRAIZUMI, Y., SANDLER, L. AND CROW, J. F. 1960. Meiotic drive in natural populations of D. melanogaster III. Populational implications of the segregation-distorter locus. Evolution, 14, 433-444.

JONES, R. N. AND REES, H. 1982. B-chromosomes. Academic Press, London.

KAYANO, H. 1961. Cytogenetic studies in Lilium callosum III. Preferential segregation of a supernumerary chromosome in EMCs. Proc. Jap. Acad., 33, 553-558.

LANDE, R. 1979. Effective deme sizes during long-term evolution estimated from rates of chromosome rearrangement. Evolution, 33, 234-251.

LANDE, R. 1985. The fixation of chromosomal rearrangements in a sub-divided population with local extinction. Heredity, 54, 323-332.

LEWIS, H. 1973. The origin of diploid neospecies in Clarkia. Amer. Nat., 107, 161-170.

SIMBERLOFF, D. S. 1974. Equilibrium theory of island biogeography. Ann. Rev. Ecol. Syst., 5, 161-182.

WENDEL, J. F., EDWARDS, M. D., STUBER, C. W. 1987. Evidence for multilocus control of preferential fertilisation in maize. Heredity, 58, 297-302.

WHITE, M. J. D. 1978. Modes of Speciation. W. H. Freeman, San Francisco.

WILBY, A. S. AND PARKER, J. S. 1986. Continuous variation in Y-chromosome structure of Rumex acetosa. Heredity, 57, 247-254.

WILBY, A. S. AND PARKER, J. S. 1987. Population structure of hypervariable Y-chrómosomes in Rumex acetosa. Heredity, $59,135-143$. 